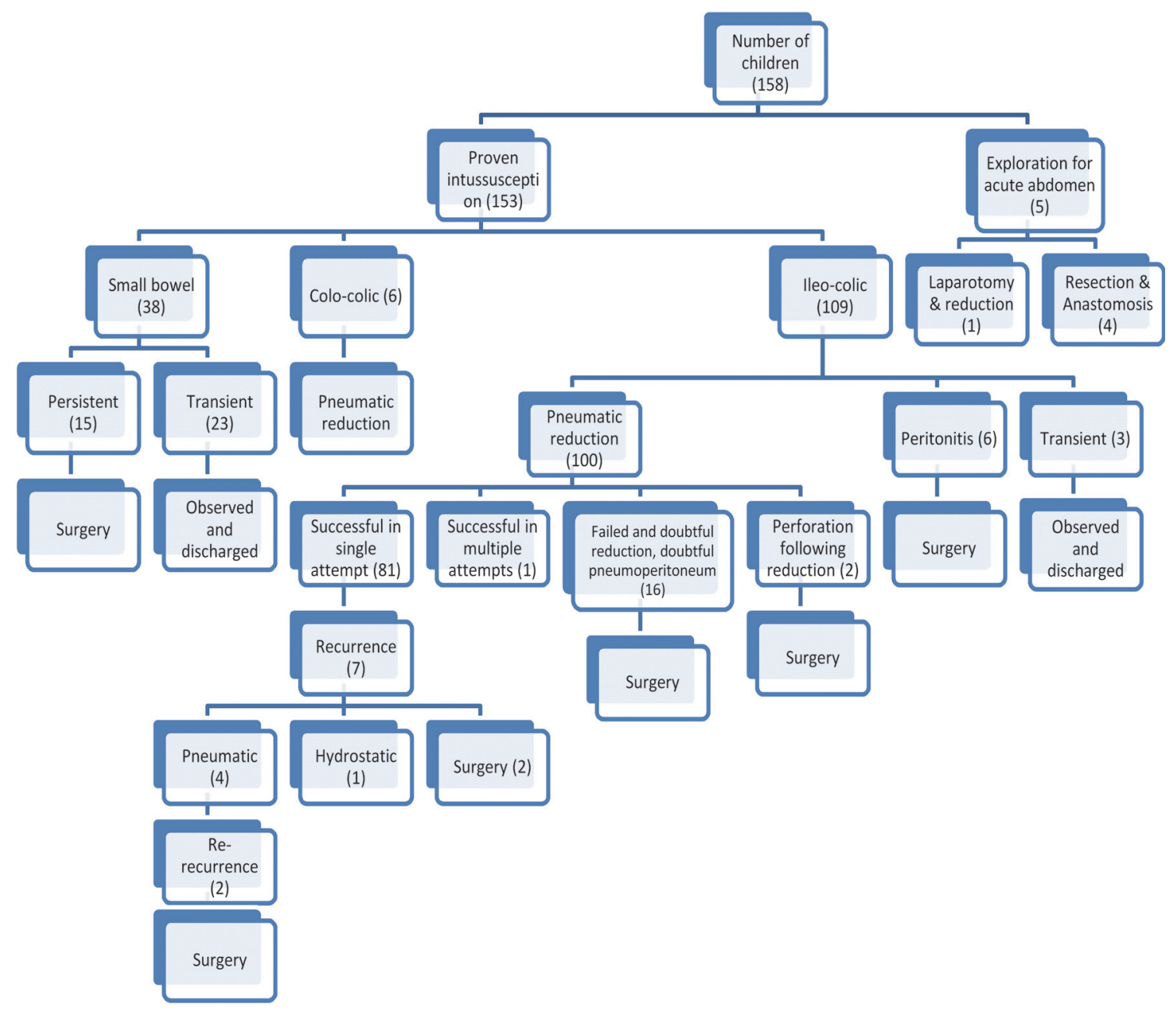

Abstract IDDF2020-ABS-0154 Figure 1 Presentation and management of intussusception

reduction. Fourteen (13\%) children with failed reduction underwent laparotomy. Twelve of these fourteen children had to undergo bowel resection (seven gangrene and five lead points) and the rest two had a successful operative reduction. Two (2\%) children with perforation following reduction, a child (1\%) each with doubtful reduction and doubtful pneumoperitoneum underwent surgery. Seven (8\%) of 89 successful reductions had a recurrence. The management of these children is depicted in figure 1.

A higher rate of failed reduction was found in children who presented at or after 48 hours of the onset of symptoms $(\mathrm{p}-0.03)$ and abdominal distension at presentation $(\mathrm{p}<0.002)$. On multiple logistic regression analysis, the children presenting at or after 48 hours onset of symptoms (OR: 11.3/p-0.039) and abdominal distension at presentation (OR: 4.46/p-0.021) were found to be associated with increased risk of failure of non-operative reduction. The variables age $<1$ year (OR:0.466/p-0.165), weight <10 kg (OR: 1.641/p-0.468), pain abdomen (OR: 1/p-0.99), vomiting (OR: 0.562/p-0.39), bilious vomiting (OR: 6.75/p-0.136), fever (OR: 2.23/p-0.357), bleeding per rectum (OR: $2.55 / \mathrm{p}-0.162)$, and palpable mass (OR: $2.74 / \mathrm{p}-0.135)$ were not associated with the failed non-operative reduction.

Conclusions The risk factors for failed non-operative reduction of intussusception include a presentation at or after 48 hours of the onset of symptoms and the presence of abdominal distension at presentation.

\section{IDDF2020-ABS-0155 A NEW DIAGNOSTIC INDEX OF SARCOPENIA FOR PREDICTING SHORT- TERM POSTOPERATIVE COMPLICATIONS IN PATIENTS UNDERGOING SURGERY FOR GASTRIC CANCER}

Xiaolong Ge*, Wei Zhou. Sir Run Run Shaw Hospital, School of Medicine, Zhejiang University, China

\subsection{6/gutjnl-2020-IDDF.100}

Background Patients with gastric cancer usually have weight loss, sarcopenia and malnutrition. Serum creatinine/cystatin C (CCR) is a new, simple, easily measured tool that could serve as a biomarker of sarcopenia. The purpose of our study was to assess the predictive ability of preoperative CCR for shortterm prognosis in patients with gastric cancer undergoing surgery.

Methods This retrospective study included 309 patients with gastric cancer undergoing surgery. Univariate and multivariate analyses were performed to identify risk factors. Patients were divided into two groups according to the optimal cut-off value of CCR. The clinical association of CCR with characteristics and postoperative complications was evaluated.

Results Among all the patients, age, lymphocyte count and cystatin C (CysC) level were independent risk factors, while red blood cell (RBC) count and CCR were independent protective factors for short-term postoperative 
complications in gastric cancer patients undergoing surgery. The preoperative CCR showed a good predictive ability for short-term postoperative complications, with an optimal cut-off value of 7.117. Patients with low CCR had a higher incidence of overall complications $(\mathrm{P}<0.001)$, including mild complications $(\mathrm{P}<0.001)$ and major complications $(\mathrm{P}<0.001)$.

Conclusions The preoperative CCR was identified as a reliable nutrition and sarcopenia assessment tool for predicting shortterm prognosis for patients with gastric cancer after surgery.

\section{IDDF2020-ABS-0159 A NEW DIAGNOSTIC INDEX OF SKELETAL MUSCLE MASS FOR PREDICTING SHORT- TERM POSTOPERATIVE COMPLICATIONS IN CROHN'S DISEASE}

Xiaolong Ge, Linna Ye, Qian Cao, Wei Zhou. Sir Run Run Shaw Hospital, School of Medicine, Zhejiang University, China

\subsection{6/gutjnl-2020-IDDF.101}

Background Skeletal muscle depletion has been suggested to affect postoperative complications in surgical patients. The creatinine-to-cystatin $\mathrm{C}$ ratio (CCR) is demonstrated as a new marker of muscle mass. The present study investigated the role of CCR in predicting postoperative complications in Crohn's disease (CD).

Methods This cohort study included 344 CD patients undergoing bowel resection from January 2016 to June 2019 at the Inflammatory Bowel Disease Center, Sir Run Run Shaw Hospital. Univariate and multivariate analyses were performed to identify risk factors for postoperative complications in CD. Receiver operating characteristic (ROC) curves were developed to examine the cutoff value of predictors in postoperative complications.

Results Among all the patients, 120 (34.9\%) had postoperative complications and $224(65.1 \%)$ recovered uneventfully. Preoperative albumin, C-reactive protein, white blood cell, creatinine, cystatin C, CCR, operative time, surgical history, laparoscopy, estimated blood loss, azathioprine and corticosteroids usage, and enteral nutrition were demonstrated to predict postoperative complications in CD (all $\mathrm{p}$ value $<0.05$ ). Additionally, higher white blood cell $(p=0.008)$, lower creatinine $(p<0.001)$, higher cystatin $C(p=0.002)$, lower CCR $(p=0.002)$, and enteral nutrition $(p=0.001)$ were identified to be independent risk factors in CD patients. CCR was identified to be with the highest area under the curve compared with other predictors, and the cutoff value was 7.804. Patients with a CCR $<7.804$ experienced more postoperative complications, including surgical site infections $(\mathrm{p}<0.001)$.

Conclusions CCR was a simple and useful predictor as evaluating skeletal muscle mass to identify patients with a high incidence of postoperative complications in CD.

\section{IDDF2020-ABS-0160 PREVALENCE AND IMPACT OF FRAILTY AMONG INFLAMMATORY BOWEL DISEASE OUTPATIENTS}

Nikola Mitrev*, Moe Nasr, Hayden Segboer, Crispin Corte, Ken Liu. AW Morrow Gastroenterology and Liver Centre, Royal Prince Alfred Hospital, Australia

10.1136/gutjnl-2020-IDDF.102
Background Frailty has been described in young patients with chronic inflammatory conditions. We aimed to determine the prevalence and impact of frailty among inflammatory bowel disease (IBD) outpatients

Methods Patients were prospectively recruited from our tertiary IBD clinic Nov 2018-Nov 2019. Frailty was defined by Fried Frailty Index (FFI). Crohn's Disease Activity Index (CDAI) for Crohn's disease (CD) or partial Mayo score for ulcerative colitis (UC) and IBD unclassified (IBDU), IBD questionnaire (IBDQ), Patient Health Questionnaire-9 (PHQ-9) and Charlson comorbidity index (CCI) were collected.

Results Forty-one patients were recruited (63\% female, median age $32,22 \%$ UC, 71\% CD, 7\% IBDU). Five patients $(12 \%)$ were frail $(\mathrm{FFI} \geq 3)$ and $36(88 \%)$ were non-frail: 20 $(49 \%)$ pre-frail $(\mathrm{FFI}=1-2), 16(39 \%)$ robust $(\mathrm{FFI}=0)$. Frail patients were older (median age $54 \mathrm{vs.31}, P=0.03$ ) with longer duration of IBD (median 20vs.6 years, $P=0.05$ ) compared to non-frail. FFI correlated with CCI (Pearson r.32, $P=0.04)$. Mean CDAI and median partial Mayo scores were higher in frail/pre-frail patients with CD (224 vs.112, $P<0.01$ ) and UC/IBDU (3vs.0.5, $P=0.03$ ) compared to robust patients. Conversely, the proportion of frail/pre-frail patients increased with IBD activity: remission (24\%), mild (36\%), moderate (89\%) and severe disease activity (100\%), $P P=0.02)$. Compared to robust patients, frail/pre-frail patients had higher mean PHQ-9 scores (11.0vs.7.1, $P=0.02)$ and higher depression rates (60\%vs.25\% with PHQ-9 $\geq 10$, $P=0.03)$. Anti-depressant use increased with FFI score $(11 \%$ $\mathrm{FFI}=0,17 \% \mathrm{FFI}=1,40 \% \mathrm{FFI}=2,100 \% \mathrm{FFI} \geq 3, P=0.02)$. Frail/pre-frail patients had lower mean IBDQ scores (141vs.169, $P=0.02$ ) compared to robust patients denoting worse quality of life (QOL).

Conclusions Frailty or prefrailty is common among IBD outpatients and associated with older age, comorbidity, IBD duration, disease activity, prednisone use, depression and lower QOL.

\section{IDDF2020-ABS-0163 REDUCED SURVIVAL POST-ENDOSCOPY FOR UPPER GASTROINTESTINAL BLEEDING IN THE COVID-19 ERA IS A SECONDARY EFFECT OF THE RESPONSE TO THE GLOBAL PANDEMIC}

${ }^{1}$ Oliver Tavabie*, ${ }^{2}$ Jennie Clough, ${ }^{3}$ Jonathan Blackwell, ${ }^{4}$ Maria Bashyam, ${ }^{5}$ Harry Martin, ${ }^{6}$ Anet Soubieres, ${ }^{6}$ Natalie Direkze, ${ }^{5}$ David Graham, ${ }^{3}$ Christopher Groves, ${ }^{7}$ Sean Preston, ${ }^{2}$ Sabina DeMartino, ${ }^{7}$ Upkar Gill, ${ }^{1}$ Bu'Hussain Hayee, ${ }^{1}$ Deepak Joshi. ${ }^{1}$ King's College Hospital, UK; ${ }^{2}$ Guy's and St Thomas' Hospital, UK; ${ }^{3}$ St George's Hospital, UK; ${ }^{4}$ St Mary's Hospital, UK; ${ }^{5}$ University College Hospital, UK; ${ }^{6}$ Imperial College Healthcare NHS Trust, UK; ${ }^{7}$ Royal London Hospital, UK

\subsection{6/gutjnl-2020-IDDF.103}

Background The COVID-19 pandemic has placed increased strain on healthcare systems worldwide with enormous reorganisation undertaken to support 'COVID-centric' services. Non-COVID-19 admissions have been shown to have reduced due to public health measures to halt viral transmission. We aimed to understand the impact of the response to the COVID-19 pandemic on the outcomes of upper gastrointestinal bleeds (UGI).

Methods A pan-London retrospective observational multicentre study comparing outcomes following endoscopy for UGI bleeds from 24th March 2020 to 20th April 2020 to the 